\title{
Role of Serum Levels of Vascular Endothelial Growth Factor and Its Receptor in Retinopathy of Prematurity
}

\author{
Binita Goswami ${ }^{1}$; Monika Goyal ${ }^{2}$; Sarita Beri ${ }^{2}$; Rajiv Garg ${ }^{2}$; Arvind Saili $^{3}$; Anju Jain ${ }^{1}$ \\ ${ }^{1}$ Department of Biochemistry, Lady Hardinge Medical College \& Associated SSK Hospital, New Delhi, India \\ ${ }_{2}^{2}$ Department of Ophthalmology, Lady Hardinge Medical College \& Associated SSK Hospital, New Delhi, India \\ 3 Department of Pediatrics, Lady Hardinge Medical College \& Associated SSK Hospital, New Delhi, India \\ *Corresponding author: Binita Goswami, Department of Biochemistry, Lady Hardinge Medical College \& Associated SSK Hospital, New Delhi, India. E-mail: binita.dr@gmail.com
}

Received: December 2, 2014; Revised: May 8, 2015; Accepted: June 25, 2015

\begin{abstract}
Background: Retinopathy of prematurity (ROP) is a disorder of developing retina of low birth weight preterm infants which can lead to blindness. One theory attributes the fibrosis seen in ROP to deregulation of vascularization in the retina. Vascular endothelial growth factor (VEGF) is one of the important mediators involved in vascularization.

Objectives: This study was carried out to assess the role of VEGF and its receptor in retinopathy of prematurity.

Patients and Methods: Around 200 preterm infants born in SSK hospital were screened at $33-34$ weeks. These babies were followed up according to the international classification of retinopathy of prematurity (ICROP) criteria. Those infants who developed ROP at 38 - 40 weeks were enrolled in group A while an equal number of infants who did not develop ROP were included in group B. Each group comprised of 30 subjects each. Venous sampling was carried out twice, once at 33 - 34 weeks and then again at 38 - 40 weeks. VEGF and VEGF-R2 were estimated by commercially available ELISA kits.

Results: There was no statistically significant difference between the levels of VEGF and VEGF-R2 in both groups at first visit as well as the follow up visit. However, the intra-group difference was significant between the first and the final visit in VEGF and VEGF-R2 levels in the cases with ROP. In the control population, the VEGF levels were significantly lower in the follow up visit as compared to the initial visit. Conclusions: Our study demonstrates that a significant difference is seen in the serum VEGF and VRGF-R2 in the second visit of the infants with ROP demonstrating that VEGF might be responsible for the initiation and aggravation of ROP.
\end{abstract}

Keywords: Retinopathy of Prematurity; VEGF; VEGFR; Angiogenesis

\section{Background}

Retinopathy of prematurity (ROP) is a disorder of developing retina of low birth weight preterm infants potentially leading to blindness in a small but significant percentage of these infants. The disease has gained more prominence in the recent years owing to the significantly lowered mortality in preterm infants. It has now become possible to resuscitate infants as young as 22 weeks due to technological advancements in neonatal care (1).

ROP is a vaso-proliferative disorder of the developing retina precipitated by increased hyper-oxygenation very common in preterm infants. As it is common knowledge, oxygen administration is a common practice in preterm infants due to its ability to improve survival prospects in such infants. However, unsupervised empiric use of oxygen led to a spurt of ROP in the late 50's (2).

Several theories were propounded to explain the etiopathogenesis of ROP. One such theory attributes the fibrosis to the dichotomy in response to hyperoxia of choroidal vessels and retinal vessels. Unlike retinal vessels, choroidal vessels cannot autoregulate under hyperoxic conditions. This leads to constriction of retinal blood vessels to the point of irreversible obliteration (3).

The process of neovascularization/angiogenesis is initi- ated and promoted by the interaction of a number of important mediators with their receptors. Some of these angiogenic factors have been subjected to intense research in the past few decades. These include fibroblast growth factor, transforming growth factor alpha, vascular endothelial growth factor (VEGF) etc. $(4,5)$.

VEGF has been implicated in ROP through studies in various mouse models. Physiological expression of VEGF in normal retina in utero likely helps in vessel development and regression. However, ex utero changes in oxygen tension may cause abnormality or down regulation of VEGF expression. Dysregulated VEGF expression may lead to untimely vaso-obliteration or exaggerated vasoproliferation $(6,7)$.

\section{Objectives}

The present study was carried out to evaluate the serum levels of VEGF and VEGF receptor 2 in premature infants with ROP.

\section{Patients and Methods}

The study was a hospital based observational comparative study carried out through multi-disciplinary collabo-

Copyright (C) 2015, Growth \& Development Research Center. This is an open-access article distributed under the terms of the Creative Commons Attribution-NonCommercial 4.0 International License (http://creativecommons.org/licenses/by-nc/4.0/) which permits copy and redistribute the material just in noncommercial usages, provided the original work is properly cited. 
ration between the departments of biochemistry, pediatrics and ophthalmology of SSK hospital. The study was commenced after prior written informed consent by the parents of the infants as well as clearance by the institutional ethical committee.

Around 200 preterm infants born in SSK hospital were screened at 33 - 34 weeks. These babies were followed up according to the international classification of retinopathy of prematurity (ICROP) criteria (8). Death during the study period led to exclusion of 5 infants. Those infants who developed ROP at 38 - 40 weeks were enrolled in group A while an equal number of infants who did not develop ROP were included in group B. Each group comprised of 30 subjects each. Venous sampling was carried out twice at 33 - 34 weeks and then again at 38 - 40 weeks. Babies with any congenital anomaly were excluded from the study. Any pulmonary disorder due to the prematurity was ruled out by clinical as well as radiological means.

Detailed antenatal history, birth history as well as postnatal history was recorded to assess the risk profile of the study group. Detailed clinical, anthropometric and ophthalmologic examination of the infants was carried out and recorded. The ophthalmic examination included pupil rigidity, corneal diameter, streak retinoscopy and indirect ophthalmoscopy.

The blood samples were processed and the serum was appropriately aliquoted and stored at - 20 degree Celsius for further analysis. VEGF was estimated by ELISA by the human VEGF-A bioLISA kit by DIACLONE. VEGF-R2 was measured by the RayBio R human VEGF-R2 ELISA kit.

\subsection{Statistical Analysis}

Statistical analysis was done with statistical package for the social science 17.0 (SPSS Inc, Chicago, Illinois). Continuous data were presented as average (minimum, maximum). Student's t test or non-parametric (Mann Whitney) analyses were used if variance was high. Paired t test was used to compare the various parameters between the two groups. The level of significance was chosen to be $\mathrm{P}<0.05$.

\section{Results}

The maternal and demographic characteristics of the study population is depicted in Table 1 . The birth weight and the gestational age is lower in the cases as well as in controls. The difference in sex distribution is striking with males predominating in the controls while females were preponderant among the cases. This may be an incidental finding. Maternal factors were assessed through history and available records. Only two factors were found to be significantly different in the cases as compared to the controls. These include oxytocin use for augmentation of labor and type of labor.

Table 2 illustrates the comparative of postnatal factors between the two groups. Factors significantly associated with ROP were use of oxygen, bag and mask, endotracheal intubation, intermittent positive pressure ventilation, Apgar score at 1 minute of birth, and acidosis.

\begin{tabular}{|c|c|c|c|}
\hline Parameters & Group A & Group B & PValue \\
\hline Birth weight, $g$ & $1064 \pm 245.45$ & $1338 \pm 196.49$ & $<0.001$ \\
\hline Gestational age, wk & $29.4 \pm 1.99$ & $31.6 \pm 1.99$ & $<0.001$ \\
\hline Male/female & $11 / 19$ & $20 / 10$ & - \\
\hline Mean age of mother, $y$ & $24.06 \pm 2.27$ & $23.43 \pm 3.21$ & 0.382 \\
\hline Any drug intake during pregnancy & $4 / 30$ & $4 / 30$ & 1.0 \\
\hline Multiple gestations, no/total & $6 / 30$ & $7 / 30$ & 0.754 \\
\hline Mean systolic blood pressure, $\mathrm{mm} \mathrm{Hg}$ & $124.13 \pm 13.24$ & $126.26 \pm 12.37$ & 0.522 \\
\hline Mean diastolic blood pressure, $\mathrm{mm} \mathrm{Hg}$ & $82.46 \pm 6.53$ & $84.4 \pm 7.71$ & 0.299 \\
\hline Diabetes, no/total & $4 / 30$ & $5 / 30$ & 0.718 \\
\hline Leaking per vaginum, no/total & $12 / 30$ & $13 / 30$ & 0.793 \\
\hline Bleeding per vaginum, no/total & $5 / 30$ & $6 / 30$ & 0.739 \\
\hline Premature rupture of membranes, no/total & $6 / 30$ & $8 / 30$ & 0.542 \\
\hline Oligo/polyhydramnios, no/total & $3 / 30$ & $2 / 30$ & 0.640 \\
\hline Syntocinon use, no/total & $8 / 30$ & $1 / 30$ & 0.011 \\
\hline Labor spontaneous/induced & $18 / 12$ & $23 / 7$ & 0.012 \\
\hline Mode of delivery- vaginal/cesarean & $27 / 3$ & $24 / 6$ & 0.278 \\
\hline Mean duration of labor, $h$ & $11.63 \pm 3.15$ & $11.67 \pm 2.88$ & 0.960 \\
\hline
\end{tabular}

a Data are presented as mean $\pm \mathrm{SD}$.

b Group A, infants with ROP; Group B, infants without ROP. 
The anthropometric and ophthalmological parameters at first visit (33-34 weeks PCA) are shown in Table 3. Mean head circumference and refraction of both eyes were significantly different in both groups.

The serum levels of VEGF and VEGF-R in the study group are summarized in Tables 4 and 5. There was no statistically significant difference between the levels of
VEGF and VEGF-R2 in both groups at first visit as well as the follow up visit. However, the intra group difference was significantly different between the first and the final visit in VEGF and VEGF-R2 levels in the cases with ROP. In the control population, the VEGF levels were significantly lower in the follow-up visit as compared to the initial visit.

Table 2. Comparison of the Post Natal Risk Factors in the Two Groups $(n=30)^{a, b}$

\begin{tabular}{lccc}
\hline Parameters & Group A & Group B & P Value \\
\hline Suctioning & $30 / 30$ & $30 / 30$ & 0.001 \\
\hline Oxygen for resuscitation & $25 / 30$ & $12 / 30$ & 0.001 \\
\hline Bag and mask & $17 / 30$ & $5 / 30$ & 0.020 \\
\hline ET and IPPV & $9 / 30$ & $2 / 30$ & 0.076 \\
\hline Drugs for resuscitation & $3 / 30$ & $0 / 30$ & 0.004 \\
\hline Apgar score at 1 min & $5.85 \pm 1.55$ & $6.86 \pm 1.10$ & 0.067 \\
\hline Apgar score at 5 min & $7.7 \pm 1.35$ & $7.93 \pm 0.78$ & 0.063 \\
\hline Ventilation & $15 / 30$ & $8 / 30$ & 0.549 \\
\hline Duration of ventilation & $24.57 \pm 30.28$ & $17.62 \pm 14.36$ & 0.071 \\
\hline Apnoiec spells & $7 / 30$ & $2 / 30$ & 0.795 \\
\hline Respiratory distress & $13 / 30$ & $14 / 30$ & 0.488 \\
\hline Surfactant & $6 / 30$ & $4 / 30$ & 0.313 \\
\hline Intracranial haemorrhage & $1 / 30$ & $0 / 30$ & 0.006 \\
\hline Acidosis & $13 / 30$ & $3 / 30$ & 0.055 \\
\hline I/v bicarbonate & $6 / 30$ & $1 / 30$ & \\
\hline Data & & & \\
\hline
\end{tabular}

a Data are presented as mean \pm SD

b Group A, infants with ROP; Group B, infants without ROP.

Table 3. Anthropometric and Ophthalmological Parameters in the Study Population $(n=30)^{a, b}$

\begin{tabular}{lccc}
\hline Parameters & Group A & Group B & P Value \\
\hline Head circumference, $\mathbf{c m}$ & $28.42 \pm 1.63$ & $29.58 \pm 1.61$ & 0.007 \\
Mid arm circum-ference, cm & $8.2 \pm 1.04$ & $8.68 \pm 1.08$ & 0.087 \\
Corneal diameter, $\mathbf{m m}$ & $9.98 \pm 0.38$ & $10.02 \pm 0.44$ & 0.757 \\
Refraction RE, diopters & $-0.78,(-4.0 \pm 0.75)$ & $-0.85,(-2.5 \pm 3.0)$ & 0.013 \\
Refraction LE, diopters & $-1.8,(-4.25 \pm 1.25)$ & $-0.76,(-3.25 \pm 2.5)$ & 0.009 \\
Pupil size, $\mathbf{m m}$ & $2.5 \pm 0.51$ & $2.5 \pm 0.51$ & 1.0 \\
\hline
\end{tabular}

a Data are presented as mean \pm SD.

b Group A, infants with ROP; Group B, infants without ROP. 
Goswami B et al.

\begin{tabular}{|c|c|c|c|}
\hline Time of Assay & Group A & Group B & P Value \\
\hline \multicolumn{4}{|l|}{ VEGF, pg/mL } \\
\hline At $32-34 \mathrm{wk}$ & $46.66 \pm 15.16$ & $55.33 \pm 25.01$ & 0.110 \\
\hline At $38-40 w k$ & $20.2 \pm 13.63$ & $24.6 \pm 11.76$ & 0.186 \\
\hline \multicolumn{4}{|l|}{ VEGF-R2, pg/mL } \\
\hline At $32-34 \mathrm{wk}$ & $7317.77 \pm 5550.3$ & $6302.27 \pm 4652.91$ & 0.446 \\
\hline At $38-40 w k$ & $5566.67 \pm 1768.17$ & $5868.33 \pm 4030.05$ & 0.709 \\
\hline \multicolumn{4}{|c|}{$\begin{array}{l}\text { a Data are presented as mean } \pm \text { SD. } \\
\text { b Group A, infants with ROP; Group B, infants without ROP. }\end{array}$} \\
\hline Parameters & First Visit & Second Visit & P Value \\
\hline \multicolumn{4}{|l|}{ Group A } \\
\hline VEGF & $46.66 \pm 15.16$ & $20.2 \pm 13.63$ & $<0.001$ \\
\hline VEGF-R2 & $7317.77 \pm 5550.3$ & $5566.67 \pm 1768.17$ & 0.077 \\
\hline \multicolumn{4}{|l|}{ Group B } \\
\hline VEGF & $55.33 \pm 25.01$ & $24.6 \pm 11.76$ & $<0.001$ \\
\hline VEGF-R2 & $6302.27 \pm 4652.91$ & $5868.33 \pm 4030.05$ & 0.710 \\
\hline
\end{tabular}

${ }^{\mathrm{a}}$ Data are presented as mean $\pm \mathrm{SD}$.

\section{Discussion}

ROP-proliferative retinopathy of premature infants is a leading cause of lifelong visual impairment among children. Researchers worldwide have been engrossed in a multitude of studies in order to ascertain the etiology behind ROP. Other than the various antenatal, natal and postnatal factors, numerous angiogenic and anti angiogenic factors are implicated in the pathogenesis of the disease like VEGF, FGF, angiopoietin among others (9). The present study was conducted to study the serum levels of VEGF A and VEGF R2 in premature infants with and without ROP and to study the correlation if any between these factors with the development of ROP.

The infants who subsequently developed ROP in our study were found to have statistically significant difference in their birth weight as well the gestational age as compared to the 'control' infants. Our findings are in accordance to the observations reported by the researchers who undertook the multi-centric trial of cryotherapy for retinopathy of prematurity. Incidence of ROP was closely correlated to earlier gestational age and low birth weight (10). Similar findings were reported by Hussain et al. who concluded from their study that no infant born after 32 weeks of gestation developed ROP (11).
The in utero maternal parameters have a pivotal role in the ex-utero life of the infant. Maternal stress in the form of systemic illnesses, drug intake, and pregnancy associated complications consequently has adverse consequences for the fetus which can hamper the normal physiological growth curve $(12,13)$. We evaluated multitude of maternal factors such as maternal age, type of delivery and pregnancy associated complications such as premature rupture of membranes, oligo/ polyhydramnios etc. We found a significant association between the incidence of ROP with the use of oxytocin and induced labor. Induced labors in a way decrease the in utero blood flow to the fetus and usher in a brief period of hypoxia. Furthermore, induction is required in pregnancies with fetal distress.

Among the post natal factors, use of oxygen, bag and mask, ET and IPPV, Apgar score at 1 minute, and acidosis were found to be statistically associated with the incidence of ROP in our study. Kim et al. associated higher incidence of ROP with ventilator use, apnea, and use of surfactant (14). The EpiBel study evaluated the co-morbidities and other risk factors associated with ROP in infants with gestational age of $<26$ weeks (15). Apgar score reflects the hypoxic state of the baby, which is an indicator of prematurity. Our finding of the implica- 
tions of Apgar score is in accordance with Ortyl et al. (16). Number of days under respiratory support was an important risk factor for ROP. Hylander et al. also reported similar findings from their study (17). The ophthalmological finding of increased incidence of myopia in the infants with ROP is in concordance with the findings of CRYO-ROP study and a similar study by Fledelius et al. (18). Shah et al. reported from their study on the incidence of ROP on VLBW babies that the risk factors for ROP are maternal preeclampsia, birth weight, and presence of pulmonary hemorrhage, duration of ventilation, and continuous positive pressure ventilation (19). In another study conducted by Filho et al. in Brazilian cohort, univariate analysis showed that gestational age (GA), BW, use of indomethacin and erythropoietin, blood transfusions, and intraventricular hemorrhage were associated with the risk of developing ROP. Logistic regression analysis showed the birth weight, gestational age, and erythropoietin therapy were the risk factors having significant odd's ratios (20).

VEGF is a member of the VEGF-PDGF family. It acts through two receptors - VEGF-R1 and VEGF-R2. VEGF-R2 is principally responsible for mediating the effects of VEGF on angiogenesis and vascular permeability (21). Different molecular pathways are involved in vasculogenesis and angiogenesis by VEGF. It acts as a mitogen for endothelial cells and mobilizes the endothelial cell precursors from the marrow. It also increases the survival of retinal endothelial cells through the inhibition of apoptosis and induces them to secrete plasminogen activator and matrix metalloproteinases. This facilitates the growth of new blood vessels (22-24). In addition it also up-regulates endothelial nitric oxide synthase which leads to increased nitric oxide levels (25).

We estimated the VEGF and VEGF-R2 levels at 33 - 34 weeks and then again at $38-40$ weeks. There was no statistically significant difference in the levels in both groups. However in the follow up sampling, there was a significant fall in VEGF levels in both groups. A similar pattern was observed for VEGF-R2 levels also. A similar study was conducted by Pieh et al. in 63 preterm infants. They also could not elicit any significant difference in the VEGF levels between cases and controls. They concluded that local instead of systemic VEGF concentrations have a role in initiating the pathogenic changes in ROP (26). This hypothesis is substantiated by a study conducted by Sonmez et al. they estimated the vitreous concentrations of VEGF in infants with ROP and compared it with normal preterm infants. A statistically significant difference in VEGF levels could be discerned between these two groups (27). Anti-VEGF therapy is also under intense investigations as many trials have raised serious doubts about its side effects. However, the BEAT-ROP trial demonstrated marked improvement in ROP with bevacizumab with preservation of peripheral retina, completion of retinal vascularization, and lower recurrence rates (28).
The limitations of our study include the small sample size, the disease was in the initial stages and the estimations were carried out in serum instead of ocular fluid. Further studies in a larger cohort of infants with ROP with a representation of all the stages of the disease with estimations in both serum as well as ocular fluid could help in understanding the role of vascular factors in the pathogenesis of retinopathy of prematurity.

\section{References}

1. Reynolds JD, Hardy RF, Palmer EA. Incidence and severity of retinopathy of prematurity. J Am Ass Ped Ophthalmol Strabis. 1999;3(5):321-2.

2. Phelps DL. Retinopathy of Prematurity: History, Classification, and Pathophysiology. Neo Rev. 2001;2(7):153e-66.

3. Chan-Ling T, Tout S, Hollander $\mathrm{H}$, Stone J. Vascular changes and their mechanisms in the feline model of retinopathy of prematurity. Invest Ophthalmol Vis Sci. 1992;33(7):2128-47.

4. Shweiki D, Itin A, Soffer D, Keshet E. Vascular endothelial growth factor induced by hypoxia may mediate hypoxia-initiated angiogenesis. Nature.1992;359(6398):843-5.

5. Pierce EA, Foley ED, Smith LE. Regulation of vascular endothelial growth factor by oxygen in a model of retinopathy of prematurity. Arch Ophthalmol.1996;114(10):1219-28.

6. Alon T, Hemo I, Itin A, Pe'er J, Stone J, Keshet E. Vascular endothelial growth factor acts as a survival factor for newly formed retinal vessels and has implications for retinopathy of prematurity. $\mathrm{Na}$ ture Med.1995;1(10):1024-8.

7. Lutty GA, McLeod DS, Merges C, Diggs A, Plouet J. Localization of vascular endothelial growth factor in human retina and choroid. Arch Ophthalmol.1996;114(8):971-7.

8. International Committee for the Classification of Retinopathy of P. The International Classification of Retinopathy of Prematurity revisited. Arch Ophthalmol. 2005;123(7):991-9.

9. Watanabe D, Suzuma K, Suzuma I, Ohashi H, Ojima T, Kurimoto M, et al. Vitreous levels of angiopoietin 2 and vascular endothelial growth factor in patients with proliferative diabetic retinopathy. Am J Ophthalmol. 2005;139(3):476-81.

10. Palmer EA, Flynn JT, Hardy RJ, Phelps DL, Phillips CL, Schaffer DB, et al. Incidence and Early Course of Retlnonathy of Prematurity. Ophthalmol.1991;98(11):1628-40.

11. Hussain N, Clive J, Bhandari V. Current incidence of retinopathy of prematurity, 1989-1997. Pediatrics. 1999;104(3):e26.

12. Wu WC, Ong FS, Kuo JZ, Lai CC, Wang NC, Chen KJ, et al. Retinopathy of prematurity and maternal age. Retina. 2010;30(2):327-31.

13. Holmström G, Thomassen P, Broberger U. Maternal risk factors for retinopathy of prematurity - a population-based study. Acta $\mathrm{Ob}$ stetricia et Gynecologica Scandinavica. 1996;75(7):628-35.

14. Kim TI, Sohn J, Pi SY, Yoon YH. Postnatal risk factors of retinopathy of prematurity. Paediatr Perinat Epidemiol. 2004;18(2):130-4.

15. Allegaert K, de Coen K, Devlieger H, EpiBel Study G. Threshold retinopathy at threshold of viability: the EpiBel study. Br J Ophthalmol. 2004;88(2):239-42.

16. Ortyl E, Lauterbach R, Starzycka M. [Retinopathy of prematurity: risk factors analysis]. Przegl Lek. 20 02;59 Suppl 1:91-4.

17. Hylander MA, Strobino DM, Dhanireddy R. ASSOCIATION OF RACE, APGAR SCORE AT FIVE MINUTES AND FORMULA FEEDING WITH RETINOPATHY OF PREMATURITY (ROP) AMONG VERY LOW BIRTH WEIGHT (VLBW) INFANTS. $† 1181$. Pediatr Res. 1997;41:199.

18. Fledelius HC. Pre-term delivery and subsequent ocular development. Acta Ophthalmol Scandinavica. 2009;74(3):297-300.

19. Shah VA, Yeo CL, Ling YL, Ho LY. Incidence, risk factors of retinopathy of prematurity among very low birth weight infants in Singapore. Ann Acad Med Singapore. 2005;34(2):169-78.

20. Fortes Filho JB, Eckert GU, Procianoy L, Barros CK, Procianoy RS. Incidence and risk factors for retinopathy of prematurity in very low and in extremely low birth weight infants in a unit-based approach in southern Brazil. Eye (Lond). 2009;23(1):25-30. 
21. Ferrara N. Vascular endothelial growth factor: basic science and clinical progress. Endocr Rev. 2004;25(4):581-611.

22. Asahara T, Takahashi T, Masuda H, Kalka C, Chen D, Iwaguro H, et al. VEGF contributes to postnatal neovascularization by mobilizing bone marrow-derived endothelial progenitor cells. EMBO J. 1999;18(14):3964-72.

23. Alon T, Hemo I, Itin A, Pe'er J, Stone J, Keshet E. Vascular endothelial growth factor acts as a survival factor for newly formed retinal vessels and has implications for retinopathy of prematurity. Nat Med.1995;1(10):1024-8.

24. Pepper MS, Ferrara N, Orci L, Montesano R. Vascular endothelial growth factor (VEGF) induces plasminogen activators and plasminogen activator inhibitor-1 in microvascular endothelial cells. Biochemic Biophysic Res Comm. 1991;181(2):902-6.

25. Dulak J, Jozkowicz A, Dembinska-Kiec A, Guevara I, Zdzien- icka A, Zmudzinska-Grochot D, et al. Nitric oxide induces the synthesis of vascular endothelial growth factor by rat vascular smooth muscle cells. Arterioscler Thromb Vasc Biol. 2000;20(3):659-66.

26. Pieh C, Agostini H, Buschbeck C, Kruger M, Schulte-Monting J, Zirrgiebel U, et al. VEGF-A, VEGFR-1, VEGFR-2 and Tie2 levels in plasma of premature infants: relationship to retinopathy of prematurity. Br J Ophthalmol. 2008;92(5):689-93.

27. Sonmez K, Drenser KA, Capone AJ, Trese MT. Vitreous levels of stromal cell-derived factor 1 and vascular endothelial growth factor in patients with retinopathy of prematurity. Ophthalmology. 2008;115(6):1065-1070 e1.

28. Azad R, Dave V, Jalali S. Use of intravitreal anti-VEGF: retinopathy of prematurity surgeons' in Hamlet's dilemma? Indian J Ophthalmol. 2011;59(6):421-2. 